\title{
Elastorrexis papulosa: una forma particular de nevo conjuntivo
}

Papular elastorrhexis: A specific form of connective tissue nevus

\section{Hugo Néstor Cabrera', María Daniela Hermida², Elba María Griffa ${ }^{3}$, María Fernanda Carriquiri'3, Graciela Sánchez ${ }^{4}$}

1. Médico dermatólogo; profesor emérito, Cátedra de Dermatología, Universidad de Buenos Aires; director, Centro Profesor Dr. Hugo Cabrera, Buenos Aires, Argentina

2. Médica dermatóloga, Centro Profesor Dr. Hugo Cabrera; Centro de Educación Médica e Investigaciones Clínicas "Norberto Quirno", CEMIC, Buenos Aires, Argentina

3. Médica dermatóloga, Centro Profesor Dr. Hugo Cabrera, Buenos Aires, Argentina

4. Médica dermatopatóloga, Centro Profesor Dr. Hugo Cabrera, Buenos Aires, Argentina.

\section{RESUMEN}

La elastorrexis papulosa es una dermatosis rara, habitualmente adquirida, caracterizada por pápulas asintomáticas, no foliculares, de color de piel normal, situada en tronco y brazos, con una histopatología que muestra intensa fragmentación de las fibras elásticas en la dermis reticular.

Se presenta un caso de elastorrexis papulosa, entidad que se clasifica entre los nevos elásticos y se hace la diferenciación diagnóstica con el síndrome de Buschke-Ollendorf.

PALABRAS CLAVE: elastorrexis papulosa; tejido conjuntivo; fibras elásticas; nevo.

\section{SUMMARY}

Papular elastorrhexis is a rare acquired dermatosis, characterized by asymptomatic, non-follicular, skin-colored papules localized on the trunk and arms. Histopathology shows important fragmentation of dermal elastic fibers.

We report a case of papular elastorrhexis and we classify this entity as an elastic nevus and differentiate it from the Buschke-Ollendorf syndrome. Key words: Papular elastorrhexis, connective tissue, elastic fibers, nevus

\section{INTRODUCCIÓN}

Se presenta el caso de un hombre de 16 años con lesiones papulosas asintomáticas congénitas, que proliferaron acentuadamente en los tres últimos años. Se localizan en la cara lateral y anterior del tronco, en los hombros y en los miembros superiores. Son de algunos milímetros de diámetro, del color de la piel normal, algunas hipocrómicas y de superficie plegada (figura 1). Algunas confluyen en elementos mayores, de varios centímetros, de aspecto verrugoso y blanquecino. En la cresta ilíaca derecha y la muñeca derecha, se observan dos lesiones de mayor tamaño, que alcanzan $1,5 \mathrm{~cm}$, redondeadas, blanquecinas y rugosas (figura 2). En el codo derecho, hay otra lesión mayor, de $2 \mathrm{~cm}$, y con superficie de aspecto atrófico. Todas las lesiones son asintomáticas.

Correspondencia:

María Daniela Hermida

Email:

mdhermida@gmail.com

Recibido: 03/04/2016

Aceptado: 18/12/2016

Conflictos de interés:

No se reportan conflictos de interés. 


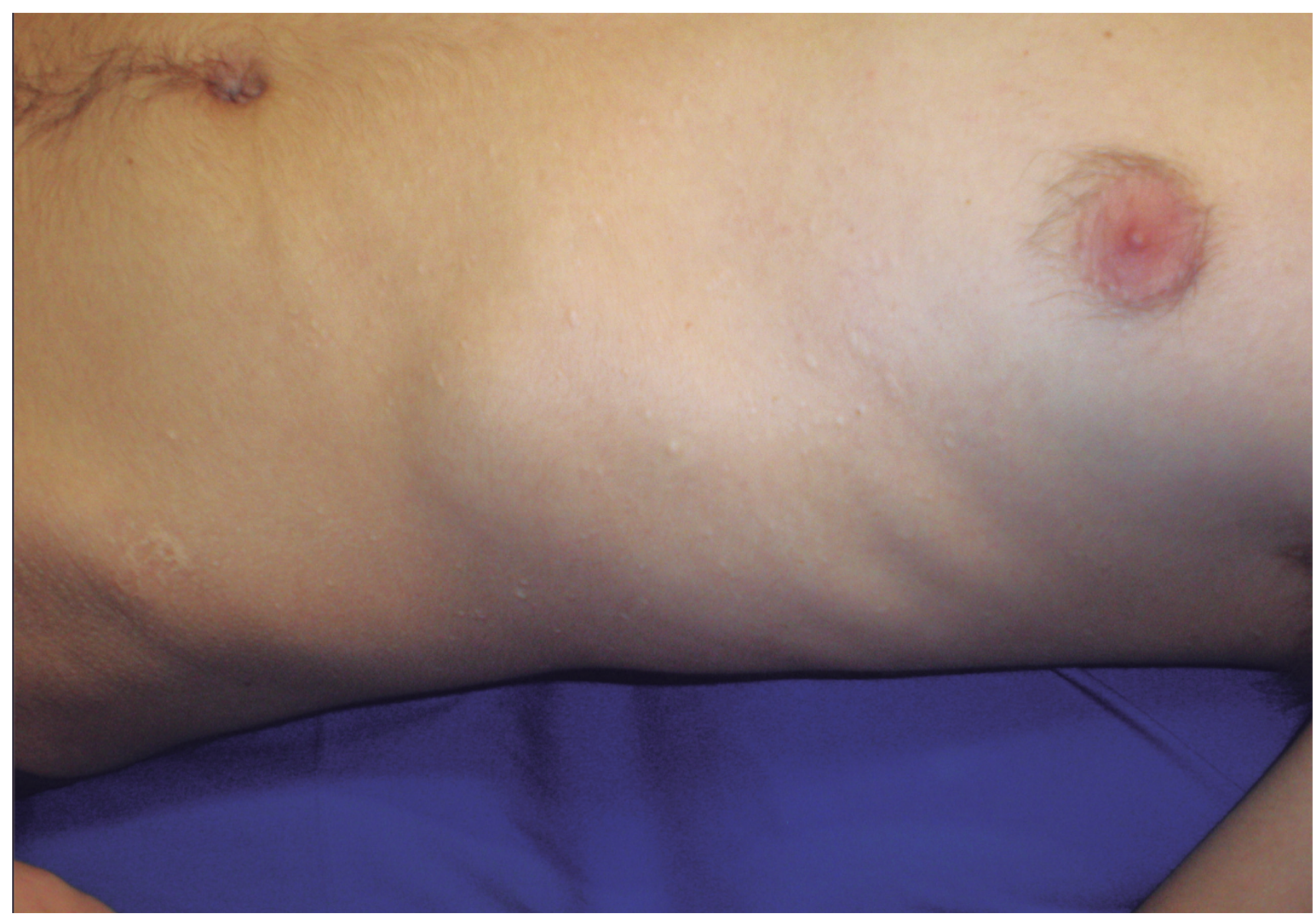

FiGURA 1. Pápulas hipocrómicas o normocrómicas en el tronco

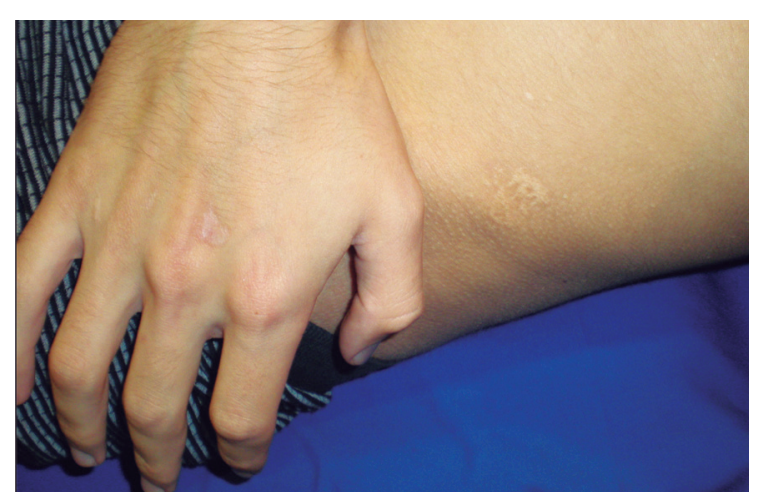

FIGURA 2. Se observan algunas lesiones de mayor tamaño en la mano y en la cadera izquierda.

El examen histológico demostró elastorrexis en todo el espesor de la dermis, con desaparición total de las fibras elásticas en algunos sectores (figura 3). El examen clínico general, los exámenes complementarios y el estudio por imágenes no mostraron alteraciones.

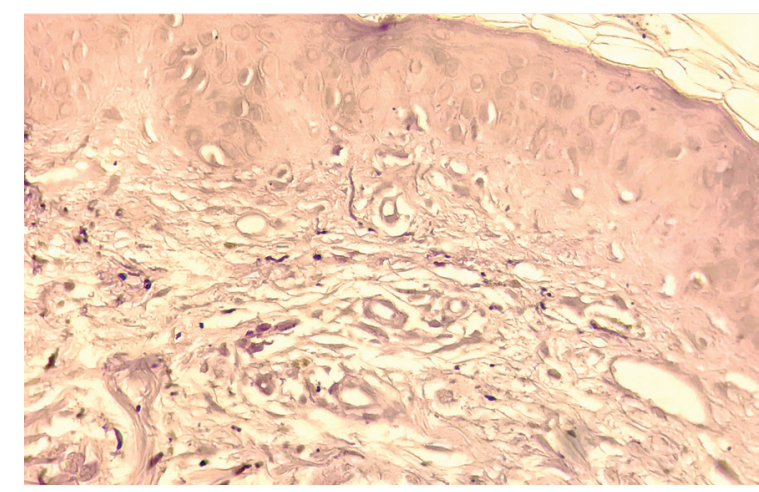

FIGURA 3. Intensa fragmentación de las fibras elásticas y desaparición de las mismas por sectores. Orceína, 100X.

\section{COMENTARIOS}

La descripción original de la elastorrexis papulosa correspondió a Bordas, et al., en $1987^{1}$, y desde entonces se han comunicado alrededor de $20 \operatorname{casos}^{2-7}$.

Los nevos del tejido conjuntivo son hamartomas constituidos predominantemente por alguno de los com- 


\section{"La elastorrexis papulosa tiene una clínica particular: pápulas de 2 a $5 \mathrm{~mm}$ de diámetro, extrafoliculares, redondas o poligonales, bien delimitadas, levemente induradas a la palpación, con superficie lisa o arrugada, con color de piel normal o hipopigmentadas, típicamente situadas en el dorso, el abdomen, los hombros, los miembros superiores y, más raramente, los dedos, el cuello y la zona mandibular"}

ponentes de la matriz extracelular: colágeno (colagenomas), elastina o glucosaminoglucanos ${ }^{8-10}$.

Los nevos del tejido elástico fueron descritos en 1921 por Lewandovsky ${ }^{11}$. Staricco y Mehregan ${ }^{12}$, en 1961, los ubicaron entre los nevos del tejido conjuntivo. Están constituidos por alteraciones de las fibras elásticas que se fragmentan, desaparecen (nevos no elásticos) o, más raramente, se condensan (elastomas). Se ven como lesiones aisladas, papulosas o en placas, o como piel de aspecto atrófico. Pueden ser solitarios o múltiples ${ }^{13}$; en esta última circunstancia, se pueden asociar a osteopoiquilosis (enfermedad de las manchas óseas), constituyendo el síndrome de BuschkeOllendorf (MIM166700), que se transmite con carácter autosómico dominante con expresión muy variable, y cuyas lesiones están presentes desde el nacimiento o aparecen en los primeros 10 años de vida. En general son elastomas, aunque también se han descrito colagenomas ${ }^{14}$. Se demostró que la pérdida de la función heterocigota en el gen LEMD3 ubicado en el cromosoma 12 q14 puede producir osteopoiquilosis, síndrome de Buschke-Ollendorf y melorreostosis ${ }^{15,16}$. Se ha descrito una variante con craneosinostosis ${ }^{17}$.

La elastorrexis papulosa tiene una clínica particular: pápulas de 2 a $5 \mathrm{~mm}$ de diámetro, extrafoliculares, redondas o poligonales, bien delimitadas, levemente induradas a la palpación, con superficie lisa o arrugada, con color de piel normal o hipopigmentadas, típicamente situadas en el dorso, el abdomen, los hombros, los miembros superiores y, más raramente, los dedos, el cuello y la zona mandibular ${ }^{18}$.

En el presente caso, además de lesiones con estas características, se aprecian algunas lesiones más grandes, blanquecinas, redondeadas y de aspecto verrugoso. La histopatología puede mostrar epidermis normal o con leve acantosis, infiltrados linfocíticos perivasculares en la dermis, leve aumento de fibras de colágeno en la dermis papilar y, con técnicas para fibras elásticas (orceína, azul victoria), se evidencia una significativa disminución y fragmentación de ellas ${ }^{19}$. Las lesiones aparecen típicamente durante la adolescencia o más tardíamente (20 años), aunque hay casos precoces (a los seis meses). El presente paciente refiere que unas pocas lesiones estaban desde el nacimiento, con una significativa aparición de nuevas lesiones a partir de los 12 años. Se afectan más frecuentemente las mujeres.

Esta entidad no tiene manifestaciones extracutáneas, de laboratorio ni radiológicas.

Se debe hacer el diagnóstico diferencial clínico con el mencionado síndrome de Buschke-Ollendorf, los colagenomas eruptivos, la dermatofibrosis lenticulares disseminata, la mucinosis papulosa y el pseudoxantoma elástico, y uno histopatológico, con las anetodermias, la elastólisis de la dermis media y, también, con el pseudoxantoma elástico.

La causa es desconocida. Las lesiones son estables. Se ha informado mejoría parcial con la infiltración de corticoides 6.

Se discute su ubicación nosológica, sobre si debe considerarse una entidad independiente o es una variante de los nevos elásticos. Nos inclinamos por esto último, considerando que es una presentación fenotípica diferente de nevos elásticos, como lo es la piel shagreen, entre los nevos conjuntivos ${ }^{20-22}$. 


\section{REFERENCIAS}

1. Bordas X, Ferrándiz C, Ribera M, Galofré E. Papular elastorrhexis: A variety of nevus anelasticus? Arch Dermatol. 1987;123:433-4.

2. Sears JK, Stone MS, Argenyi Z. Papular elastorrhexis. A variant of connective tissue nevus. Case reports and review of the literature. J Am Acad Dermatol. 1988;19:409-14.

3. Schirren H, Schirren CG, Stolz W, Kind P, Plewig G. Papular elastorrhexis: A variant of dermatofibrosis lenticularis disseminate (Buschke-Ollendorf syndrome)? Dermatology. 1994;189:368-72.

4. Buechner SA, Itin P. Papular elastorrhexis: Report of five cases. Dermatology. 2002;205:198-200.

5. Ryder HF, Antaya RJ. Nevus anelasticus, popular elastorrhexis, and eruptive collagenoma: Clinically similar entities with focal absence of elastic fibers in childhood. Pediatric Dermatol. 2005;22:153-7.

6. Lee SH, Park SH, Song KY, Yoon TJ, Kim TH. Papular elastorrhexis in childhood improved by intralesional injections of triamcinolone. J Dermatol. 2001;28:569-71.

7. Pajot C, Le Clec'h C, Houreau F, Croue A, Verret JL. Élastorrhexie papuleuse: deux cas. Ann Dermatol Venereol. 2008;135:757-61.

8. Cabrera H, García S. Nevos. Buenos Aires: Editorial Actualizaciones Médicas; 1998.

9. McClung AA, Blumberg MA, Huttenbach Y, Colome-Grimmer MI, Raimer SS. Development of collagenomas during pregnancy. J Am Acad Dermatol. 2005;53:S150-3.

10. Uitto J, Santa Cruz DJ, Eisen AZ. Connective tissue nevi of the skin. Clinical, genetic, and histopathologic classification of hamartomas of the collagen, elastin, and proteoglycan type. J Am Acad Dermatol. 1980;3:441-61.

11. Lewandowsky F. Über einem eigentumlichen nevus der brustgegend. Arch Dermatol Syphilol. 1921;131:90-4.

12. Staricco RG, Mehregan AH. Nevus elasticus and nevus elasticus vascularis. Arch Dermatol. 1961;84:943-7.

13. Crivellato E. Disseminated nevus anelasticus. Int J Dermatol. 1986;25:171-3.

14. Cabrera HN. Nevos conectivos. Su asociación con osteopoiquilosis. Arch Argent Dermatol. 1977;1:3-11.

15. Hellemans J, Preobrazhenska O, Willaert A, Debeer P, Verdonk PC, Costa T, et al. Loss-of function mutations in LEMD3 result in osteopoikilosis, Buschke-Ollendorf syndrome and melorheostosis. Nat Genet. 2004;36:1213-8.

16. Yuste-Chaves M, Cañueto J, Santos-Briz Á, Ciria S, González Sarmiento R, Unamuno P. Phenotypic variation resulting from a novel c.2203C $>$ T nonsense mutation in LEMD3. Pediatr Dermatol. 2011;28:447-50.

17. Reid EM, Baker BL, Stees MA, Stone SP. Buschke-Olelendorf syndrome: A 32-month-old boy with elastomas and craniosynostosis. Pediatr Dermatol. 2008;25:349-51.

18. Tan C, Zhu WY, Min ZS. Papular elastorrhexis located on occipito-cervical and mandibular regions. Eur J Dermatol.
2009;19:399-400

19. Flores PB, Cuevas J, Sánchez C, De Eusebio E, Vergara A. Papular elastorrhexis: An acquired disorder of elastic tissue. Eur J Dermatol. 2010;20:525-6.

20. Del Pozo J, Martínez W, Sacristán F, Fernández-Jorge B, Fonseca E. Papular elastorrhexis, a distinctive entity? Am J Dermatopathol. 2008;30:188-90.

21. Lewis K, Bercovitch L, Dill SW, Robinson-Bostom L. Acquired disorders of elastic tissue. Part II. Decreased elastic tissue. J Am Acad Dermatol. 2004;51:165-85.

22. Garg T, Chander R, Jain A, Nangia A, Mal J. Multiple elastomas in a 6-month-old child. Indian J Dermatol Venereol Leprol. 2010;76:578-9. 